\title{
Investigation of Association with First-Trimester Free Human Chorionic Gonadotropin - $\beta$, Pregnancy - Associated Plasma Protein-A, and Adverse Pregnancy Outcomes
}

\author{
Gokhan GONEN1, Yasam Kemal AKPAK², Murat MUHCU³ \\ Ankara, Turkey
}

\begin{abstract}
OBJECTIVES: This study aimed to investigate the association between first-trimester screening maternal serum markers (free human chorionic gonadotropin-beta ( $\beta-\mathrm{hCG}$ ), pregnancy-associated plasma protein-A, and adverse pregnancy outcomes (gestational hypertension, preeclampsia, preterm delivery, intrauterine growth restriction, oligohydramnios, intrauterine ex-fetus, abruptio placentae, and gestational diabetes mellitus).
\end{abstract}

STUDY DESIGN: This was a retrospective cohort study including 1516 women who delivered a singleton pregnancy at GATA Haydarpasa Education Hospital from 2006 to 2009. Patients with multiple pregnancies, chromosome aberrations, or fetal anomalies were excluded. Extreme values of correctedpregnancy-associated plasma protein-A and free $\beta-\mathrm{hCG}$, and their association with adverse pregnancy outcomes were analyzed.

RESULTS: Adverse pregnancy outcomes at the cutoff level of $\leq 0.25$ c-pregnancy-associated plasma protein-A multiples of median values positive likelihood ratio (+LR) was $7.5(95 \% \mathrm{Cl} 2.426-19.836)$ and had a significant correlation $(r=0.108, p<0.01)$. It was also a significant correlation with adverse pregnancy outcomes $(r=0.189, p<0.01)$ at the cut-off level of $\leq 0.50$ corrected-pregnancy-associated plasma protein-A multiples of median values and the +LR was $2.388(95 \% \mathrm{Cl} 1.889-2.991)$. Association between low corrected-pregnancy-associated plasma protein-A multiples of median values and adverse pregnancy outcomes were statistically significant and had a poor association (area under the curve, 0.630 $95 \% \mathrm{Cl} 0.596-0.663, p<0.01)$. Preeclampsia was statistically significant, however had a fair association (area under the curve, $0.7495 \% \mathrm{Cl} 0.690-0.802, p<0.01$ ). Preterm birth was statistically significant but had a poor association (area under the curve, $0.56895 \% \mathrm{Cl} 0.512-0.624, p<0.05$ ).

CONCLUSION: First-trimester maternal serum low pregnancy-associated plasma protein-A values are significantly associated with adverse pregnancy outcomes. It may be a useful tool to predictive not only chromosome anomalies but also adverse pregnancy outcomes.

Keywords: Fetal growth restriction, Gestational hypertension, Human chorionic gonadotrophin beta subunit, Nuchal translucency, Preeclampsia, Pregnancy-associated plasma protein A

\section{Gynecol Obstet Reprod Med 2022;28(1):7-15}

${ }^{1}$ Department of Obstetrics and Gynecology, Sehit Sait Erturk State Hospital, Ankara, Turkey

${ }^{2}$ Department of Obstetrics and Gynecology, University of Health Sciences Tepecik Training and Research Hospital, Izmir, Turkey

${ }^{3}$ Department of Obstetrics and Gynecology, University of Health Sciences, Umraniye Training and Research Hospital, Istanbul, Turkey

Address of Correspondence: Gokhan Gonen

Department of Obstetrics and Gynecology, Sehit Sait Erturk State Hospital, 06790

Ankara, Turkey

gokhangonen2002@yahoo.com

Submitted for Publication: 10.10.2020 Revised for Publication: 16.10.2020 Accepted for Publication: 05.02.2021 Online Published: 20.04.2021

ORCID IDs of the authors: $\quad$ GG:0000-0002-8271-3826

YKA: 0000-0002-1699-8667, MM: 0000-0001-7039-375X

\begin{tabular}{c|c}
\hline Quick Response Code: & Access this article online \\
\cline { 2 - 2 } & $\begin{array}{l}\text { Website: www.gorm.com.tr } \\
\text { e- mail: info@gorm.com.tr }\end{array}$ \\
\cline { 2 - 2 } & DOI:10.21613/GORM.2021.1158 \\
\hline
\end{tabular}

\section{Introduction}

First-trimester screening tests were found to be effective for trisomy 21 screening (1-5). This screening technique is used to evaluate fetal nuchal translucency (NT) ultrasound and maternal serum markers pregnancy-associated plasma protein-A (PAPPA) and free human chorionic gonadotropin beta subunit ( $\beta$-hCG) (6-8). The insulin-like growth factor (IGF) system plays an important role in regulating ovarian follicular development (ovarian follicle growth, selection, atresia, cellular differentiation, oocyte maturation, cumulus expansion, and follicle dynamics), and steroidogenesis. PAPPA is a secreted metalloprotease responsible for the cleavage of IGF binding protein-4 in the ovary $(8,9)$. These data demonstrate an im-

How to cite this article: Gonen G. Akpak YK. Muhcu M. Investigation of Association with First-Trimester Free Human Chorionic Gonadotropin - $\beta$, Pregnancy - Associated Plasma Protein-A, and Adverse Pregnancy Outcomes Gynecol Obstet Reprod Med. 2022;28(1):7-15 
portant role of PAPPA in modulating ovarian function, placentation, placental function, and female fertility by control of the bioavailability of ovarian $\operatorname{IGF}(8,10)$. If the level of PAPPA is insufficient to successfully cleave IGF, IGF remains in its bound, inactive form, possibly leading to diminished fetal and placental growth (11).

While several other publications have shown statistically significant associations between low PAPPA levels and preeclampsia, fetal loss, preterm birth, and (IUGR), they have failed to demonstrate high sensitivity and high positive predictive values to deem a patient high or low risk for the adverse outcomes (12-20). Free $\beta$-hCG is a syncytiotrophoblastderived promoter of cell growth and differentiation in the embryo (21). Evidence is lacking to support an association between free $\beta$-hCG and non-chromosomal adverse pregnancy outcomes $(19,22,23)$.

In this retrospective cohort study, we aimed to investigate the relationship between the first-trimester screening maternal serum markers especially corrected PAPPA levels, and adverse pregnancy outcomes such as gestational hypertension, preeclampsia, IUGR, oligohydramnios, preterm birth, intrauterine ex fetus (IUEF), gestational diabetes mellitus (GDM), placental abruption.

\section{Material and Method}

This was a retrospective cohort study including the final number of 1516 women delivered of a singleton pregnancy at GATA Haydarpasa Education Hospital from 2006 to 2009 for whom first-trimester serum markers analysis, as well as pregnancy outcome data, were available. Multiple pregnancies confirmed fetal chromosomal abnormalities and fetal demise before 20 weeks of gestation were excluded. Records with incomplete pregnancy outcome data were excluded. Clinical records and hospital computer automation systems were used as data sources. The three first-trimester markers including PAPPA level, free $\beta$-hCG, and NT were evaluated. Information on maternal medical and obstetrical history, gestational age, maternal weight, fetal crown-rump length (CRL), and NT measures at the examination, type of delivery, time of weeks at delivery, and pregnancy outcomes were collected. The adverse pregnancy outcomes examined including gestational hypertension (GHT), preeclampsia, preterm birth, IUGR, oligohydramnios, low birth weight, IUEF, abruptio placentae, and GDM.

Pregnancy-associated plasma protein-A values and free $\beta$ hCG values measured in maternal serum were obtained by the chemiluminescence method using immulite kits in Immulite 1000 analyzer of the Hospital Biochemistry Laboratory. Multiples of median values (MOM) of the corrected median were calculated by using the first-trimester screening test form which was filled in perinatology outpatient clinic with PRISCA package software program. Demographic distribu- tions of patients, ultrasonographic findings in first-trimester between 10 weeks 0 days and 13 weeks 6 days of gestation (CRL between 38 and $83 \mathrm{~mm}$ ), and biochemical test data were collected in the Office 2007 Excel program.

Preterm birth was defined as a delivery occurring before 37 weeks of pregnancy. IUGR was defined as a fetus whose estimated weight was below the $10^{\text {th }}$ percentile for gestational age. GHT was defined as a new onset of hypertension (systolic blood pressure $140 \mathrm{mmHg}$ and/or diastolic blood pressure 90 $\mathrm{mmHg}$ ) were documented on at least two occasions at least 6 hours apart at 20 weeks of gestation, in the absence of proteinuria. Preeclampsia was defined as the presence of systolic blood pressure $\geq 140 \mathrm{mmHg}$ and/or diastolic blood pressure $\geq 90 \mathrm{mmHg}$ after 20 weeks of pregnancy detected on two or more occasions separated by $6 \mathrm{~h}$ with the presence of proteinuria randomly detection of $\geq 2+$ on dipstick or $0.3 \mathrm{~g}$ in a $24 \mathrm{~h}$ urine specimen. Intrauterine fetal demise, ex fetus, or stillbirth was defined as intrauterine fetal deaths after 20 weeks of pregnancy. Oligohydramnios was defined as an amniotic fluid index $<5 \mathrm{~cm}$. GDM was diagnosed if fasting glucose level 92 and $126 \mathrm{mg} / \mathrm{dL}$ at first-trimester or at least one value of plasma glucose concentration 92, 180, and $153 \mathrm{mg} / \mathrm{dL}$ (for fasting, 1and 2-h post-glucose load glucose values, respectively), after performing a $75 \mathrm{~g}$ oral glucose tolerance test or at least two values of plasma glucose concentration 95, 180, 155, and 140 $\mathrm{mg} / \mathrm{dL}$ (for fasting, 1., 2., and 3. hour post-glucose load glucose values, respectively), after performing a $100 \mathrm{~g}$ oral glucose tolerance test if $50 \mathrm{~g}$ oral glucose tolerance test first hour glucose level was $>140 \mathrm{mg} / \mathrm{dL}$ at $24-28$ weeks of gestation. Small for gestational age (SGA) was defined as a birth weight of less than the $10^{\text {th }}$ percentile for gestational age.

In the primary analysis, relations between $\leq 0.25 \mathrm{MOM}$ and $\leq 0.50$ MOM levels of PAPPA and adverse pregnancy outcomes were evaluated. Descriptive analysis of adverse pregnancy outcomes under $<1.0 \mathrm{MOM}$ level with $0.25 \mathrm{MOM}$ intervals of PAPPA was performed (Figure 1).

Statistical analyses were performed with the SPSS package (SPSS Inc., Windows, version 17.0). Categorical variables were compared by the $\chi^{2}$ test. Continuous variables were described using median and interquartile ranges and categorical variables using frequencies. Spearman correlation and Receiver operating characteristic (ROC) curve analysis were performed to evaluate the relationship between independent variables. The traditional academic point system was used to classify the area under the curve (AUC). AUC from 0.90 to $1.0,0.80$ to $0.90,0.70$ to $0.80,0.60$ to 0.70 , and 0.50 to 0.60 indicates excellent, good, fair, poor, and fail association ability. Likelihood ratio, sensitivity, specificity, predictive values, and 95\% CI were calculated by using the diagnostic test calculator program of evidence-based medicine toolbox in (https://ebmtools.knowledgetranslation.net/calculator/diagnostic/) webpage. Statistical significance was defined as $p$-value 0.05 . 


\begin{tabular}{|c|c|c|}
\hline & Adverse pregnancy outcomes & Normal pregnancy outcomes \\
\hline$>1.0$ & $16(16.2 \%)$ & $517(83.8 \%)$ \\
\hline $0.75-1.0$ & $61(20.5 \%)$ & $237(79.5 \%)$ \\
\hline $0.50-0.75$ & $103(28.1 \%)$ & $263(71.9 \%)$ \\
\hline $0.25-0.50$ & $90(41.1 \%)$ & $129(58.9 \%)$ \\
\hline$\leq 0.25$ & $11(68.7 \%)$ & $129(58.9 \%)$ \\
\hline
\end{tabular}

Figure 1: Corrected PAPPA MOM values, percentile distribution of adverse pregnancy outcomes at $0.25 \mathrm{MOM}$ intervals below 1.0 MOM, $n=1516$, Istanbul 2006-2009. Corrected PAPPA MOM values mean: pregnancy-associated plasma protein-A multiples of the median values of the corrected median that was calculated by using the first-trimester screening perinatology outpatient clinic test form with PRISCA package software program, Gray color in each bar shows the number and percentage of adverse pregnancy outcomes (gestational hypertension, preeclampsia, preterm delivery, fetal growth restriction, oligohydramnios, intrauterine ex fetus, abruptio placentae, and gestational diabetes mellitus). White areas in each bar show the number and percentage of normal pregnancy outcomes.

\section{Results}

The final number of the study population was 1516 after excluding multiple pregnancies, fetal chromosomal abnormalities, and fetal demise before 20 weeks of gestation, records with incomplete pregnancy outcome data during the study period, from the beginning of January 2006 to at the end of December 2008. Descriptive characteristics of the study group of 1516 pregnant women were shown in table I.

Adverse pregnancy outcomes among 1516 pregnant women were as follows $33(2.2 \%)$ GHT, 68 (4.5\%) preeclampsia, 107 (7.1\%) preterm birth, 29 (1.9\%) IUGR, 14 (0.9\%) IUEF, 33 (2.2\%) oligohydramnios, 69 (4.6\%) GDM, $12(0.8 \%)$ abruptio placentae. Adverse pregnancy outcomes distribution showed among 0.25 MOM intervals of under $<1.0$ c-PAPPA MOM values (Table II). It has been used maternal weight correction of maternal serum PAPPA values to reduces the population variability of the markers and the impact on detection rate and false-positive rate is very small (3-5). Corrected PAPPA MOM was equal to PAPPA MOM/ ((79.858×1/weight) -0.230$)$.

Table I: Patients characteristics of the study group, Istanbul 2006-2009 all ( $n=1516)$

\begin{tabular}{lllll}
\hline Maternal age & 29.01 & 4.467 & 19 & 43 year \\
Maternal weight & 61.00 & 8.683 & 41 & $705 \mathrm{~kg}$ \\
Gestational age at the sampling day & 87.76 & 4.10 & 73 & 97 days \\
CRL & 63.00 & 7.982 & 0.10 & 5.08 \\
corrected PAPPA MOM & 0.87 & 0.57 & 0.17 & 6.51 \\
Free $\beta$-hCG MOM & 0.88 & 0.86 & 0.11 & 3.16 \\
NT MOM & 0.96 & 0.22 & 500.00 & 5150.00 gram \\
Birth weight & 3350.00 & 545.72 & 22 & 42 week \\
Gestational age of weeks at delivery & 38.38 & 2.14 & 20 & \\
\hline
\end{tabular}

CRL: Crown-rump length, PAPPA: Pregnancy-associated plasma protein-A, MOM: Multiples of median values, $\beta$-hCG: Human chorionic-gonadotropin beta, NT: Nuchal translucency, data are given as median, standard deviation, minimum, and maximum values

Table II: Corrected pregnancy-associated plasma protein-A, multiples of median values and frequency distribution of adverse pregnancy outcomes at 0.25 multiples of median values intervals below 1.0 multiples of median values, Istanbul 2006-2009.

\begin{tabular}{|c|c|c|c|c|c|c|}
\hline \multirow[b]{2}{*}{ Adverse pregnancy outcomes } & \multicolumn{5}{|c|}{ Corrected PAPPA MOM levels } & \multirow[b]{2}{*}{ Total } \\
\hline & $\leq 0.25$ & $0.25-0.50$ & $0.51-0.75$ & $0.75-1.0$ & $>1.0$ & \\
\hline Gestational hypertension & $2(6.1 \%)$ & $8(24.2 \%)$ & $7(21.1 \%)$ & $7(21.2 \%)$ & $9(27.3 \%)$ & 33 \\
\hline Preeclampsia & $2(2.9 \%)$ & $25(36.8 \%)$ & $25(36.8 \%)$ & $7(10.3 \%)$ & $9(13.2 \%)$ & 68 \\
\hline Preterm birth & $4(3.8 \%)$ & $20(19.2 \%)$ & $25(23.4 \%)$ & $22(21.2 \%)$ & $36(33.6 \%)$ & 107 \\
\hline Intrauterine growth restriction & $2(6.9 \%)$ & $9(31.0 \%)$ & $9(31.0 \%)$ & $5(17.2 \%)$ & $4(13.8 \%)$ & 29 \\
\hline Oligohydramnios & $0(0.0 \%)$ & $5(15.2 \%)$ & $11(33.3 \%)$ & $6(18.2 \%)$ & $11(33.3 \%)$ & 33 \\
\hline Abruptio placentae & $0(0.0 \%)$ & $1(\% 8.3)$ & $4(\% 33.3)$ & $2(\% 16.7)$ & $5(\% 41.7)$ & 12 \\
\hline Fetal demise & $0(0.0 \%)$ & $5(35.7 \%)$ & $4(28.6 \%)$ & $2(14.3 \%)$ & $3(21.4 \%)$ & 14 \\
\hline Gestational diabetes mellitus & $1(1.4 \%)$ & $17(14.6 \%)$ & $18(26.1 \%)$ & $10(14.5 \%)$ & $23(33.3 \%)$ & 69 \\
\hline Total adverse pregnancy outcome & $11(3.0 \%)$ & $90(24.7 \%)$ & $103(28.2 \%)$ & $61(16.7 \%)$ & $100(27.4 \%)$ & 365 \\
\hline Study Population & $16(1.1 \%)$ & $219(14.4 \%)$ & $366(24.1 \%)$ & $298(19.7 \%)$ & $617(40.7 \%)$ & 1516 \\
\hline
\end{tabular}

PAPPA: Pregnancy-associated plasma protein-A, MOM: Multiples of median values, 
There was no relationship between high c- PAPPA or free corrected $\beta$-hCG MOM levels and adverse pregnancy outcomes except it was a statistically significant association with the cut-off level of corrected free $\beta$-hCG $\geq 2.9$ MOM values and fetal macrosomia $(>4500 \mathrm{~g})(\mathrm{r}=0.068, p<0.01)$. Also, we analyzed that below the $5 \mathrm{t}^{\mathrm{h}}$ percentile of corrected free $\beta$-hCG $(\leq 0.33 \mathrm{MOM})$ cutoff level, it was evidence of statistically significant association with small for gestational age $\left(10^{\text {th }}\right.$ percentile birth weight $)(\mathrm{r}=0.088), p<0.01)$. Corrected PAPPA MOM values frequency distribution and percentiles of adverse pregnancy outcomes were shown in figure 1 and Table II.

When the number of adverse pregnancy outcomes analyzed by One-Sample Kolmogorov-Smirnov test whether it is appropriate to a normal distribution, groups have been found to follow a normal distribution $(\mathrm{p}<0.05)$. Nonparametric tests $\chi^{2}$ and Spearman correlation were used for correlation analysis for data analysis.

The likelihood ratio was preferred to sensitivity, specificity, and predictive values. Because they have advantages over sensitivity, specificity, and positive/negative predictive values because they are less likely to change with the prevalence of the disorder, they can be calculated for several levels of the symptom/sign or test, they can be used to combine the results of multiple diagnostic tests and they can be used to calculate the post-test probability for a target disorder.

The association with corrected PAPPA MOM values under or equal to $0.25 \mathrm{MOM}$ and adverse pregnancy outcomes were shown in table III. Two gestational hypertension cases $(6.1 \%)$ were detected at the cut off level of $\leq 0.25$ c-PAPPA
MOM values. The positive likelihood ratio (+LR) was 6.778 (95\%CI 1.52-27.12) and had a statistically significant correlation at this cut off value $(\mathrm{r}=0.073, p<0.01)$. Two preeclampsia cases $(2.9 \%)$ were detected at the cutoff level of $\leq 0.25 \mathrm{c}-$ PAPPA MOM values. The positive likelihood ratio was 2.9 (95\% CI 0.705-13.118) but had no statistically significant correlation at this cutoff value $(\mathrm{r}=0.040, p>0.05)$. Four Preterm birth cases $(3.8 \%)$ were detected at the cutoff level of $\leq 0.25 \mathrm{c}$ PAPPA MOM values. The positive likelihood ratio (+LR) was 4.111 (95\% CI 1.44-13.378) and had a statistically significant correlation at this cutoff value $(\mathrm{r}=0.072, p<0.01)$. Two IUGR cases $(6.9 \%)$ were detected at the cutoff level of $\leq 0.25$ cPAPPA MOM values. Positive likelihood ratio (+LR) was 7.667 (95\%CI 1.744-30.772) and had statistically significant correlation $(\mathrm{r}=0.80, p<0.01)$. One GDM case $(1.4 \%)$ was detected at the cutoff level of $\leq 0.25$ c-PAPPA MOM values. The positive likelihood ratio was 1.4 (95\%CI 0.187-10.432) and had no statistically significant correlation $(\mathrm{r}=0.008, \mathrm{p}>0.05)$. Oligohydramnios, abruptio placentae, IUEF had not any cases at the cutoff level of $\leq 0.25$ c-PAPPA MOM values. Total adverse pregnancy outcome had 11 cases $(3.0 \%)$ at the cutoff level of $\leq 0.25$ c-PAPPA MOM values. The positive likelihood ratio was 7.5 (95\%CI 2.426-19.836) and had a statistically significant correlation $(\mathrm{r}=0.108, p<0.01)$ (Table III).

Association with cutoff level of $\leq 0.50$ c-PAPPA MOM values and adverse pregnancy outcomes were showed in table IV. It was a statistically significant correlation with GHT $(\mathrm{r}=0.61, p<0.05)$, preeclampsia $(\mathrm{r}=0.145, p<0.01)$, preterm birth $(\mathrm{r}=0.053, p<0.05)$, IUGR $(\mathrm{r}=0.087, p<0.01)$, IUEF $(\mathrm{r}=0.054, p<0.05)$, GDM $(\mathrm{r}=0.064, p<0.05)$, total adverse

Table III: Association with corrected pregnancy-associated plasma protein-A, multiples of median cutoff values of $\leq 0.25$ multiples of median values and adverse pregnancy outcomes

\begin{tabular}{|c|c|c|c|c|c|c|c|c|c|}
\hline & $n=$ & $\begin{array}{l}\text { Sensitivity } \\
95 \% \mathrm{Cl}\end{array}$ & $\begin{array}{l}\text { Specificity } \\
95 \% \mathrm{Cl}\end{array}$ & $\begin{array}{l}\text { PPV } \\
95 \% \mathrm{Cl}\end{array}$ & $\begin{array}{l}\text { NPV } \\
95 \% \mathrm{Cl}\end{array}$ & $\begin{array}{l}+\mathrm{LR} \\
95 \% \mathrm{CI}\end{array}$ & \begin{tabular}{|l|}
- LR \\
$95 \% \mathrm{CI}$
\end{tabular} & $r$ & $\mathbf{p}$ \\
\hline $\begin{array}{l}\text { Gestational } \\
\text { hypertension }\end{array}$ & $\begin{array}{l}2 \\
(6.1 \%)\end{array}$ & $\begin{array}{l}0.061 \\
(0.017-0.196)\end{array}$ & $\begin{array}{l}0.991 \\
(0.984-0.994)\end{array}$ & $\begin{array}{l}0.125 \\
(0.035-0.36)\end{array}$ & $\begin{array}{l}0.979 \\
(0.971-0.985)\end{array}$ & $\begin{array}{l}6.778 \\
(1.52-27.12)\end{array}$ & $\begin{array}{l}0.948 \\
(0.869-1.034)\end{array}$ & 0.073 & $p<0.01$ \\
\hline Preeclampsia & $\begin{array}{l}2 \\
(2.9 \%)\end{array}$ & $\begin{array}{l}0.029 \\
(0.008-0.101)\end{array}$ & $\begin{array}{l}0.99 \\
(0.984-0.994)\end{array}$ & $\begin{array}{l}0.125 \\
(0.035-0.36)\end{array}$ & $\begin{array}{l}0.956 \\
(0.944-0.965)\end{array}$ & $\begin{array}{l}2.9 \\
(0.705-13.118)\end{array}$ & $\begin{array}{l}0.981 \\
(0.94-1.022)\end{array}$ & 0.040 & $p>0.05$ \\
\hline Preterm birth & $\begin{array}{l}4 \\
(3.8 \%)\end{array}$ & $\begin{array}{l}0.037 \\
(0.015-0.092)\end{array}$ & $\begin{array}{l}0.991 \\
(0.985-0.995)\end{array}$ & $\begin{array}{l}0.25 \\
(0.102-0.495)\end{array}$ & $\begin{array}{l}0.931 \\
(0.917-0.943)\end{array}$ & $\begin{array}{l}4.111 \\
(1.44-13.378)\end{array}$ & $\begin{array}{l}0.972 \\
(0.935-1.008)\end{array}$ & 0.072 & $p<0.01$ \\
\hline $\begin{array}{l}\text { Intrauterine growth } \\
\text { restriction }\end{array}$ & $\begin{array}{l}2 \\
(6.9 \%)\end{array}$ & $\begin{array}{l}0.069 \\
(0.019-0.22)\end{array}$ & $\begin{array}{l}0.991 \\
(0.984-0.984)\end{array}$ & $\begin{array}{l}0.125 \\
(0.035-0.36)\end{array}$ & $\begin{array}{l}0.982 \\
(0.974-0.988)\end{array}$ & $\begin{array}{l}7.667 \\
(1.744-30.772)\end{array}$ & $\begin{array}{l}0.939 \\
(0.851-1.038)\end{array}$ & 0.80 & $p<0.01$ \\
\hline Oligohydramnios & 0 & - & - & - & - & - & - & & \\
\hline Abruptio placentae & 0 & - & - & - & - & - & - & & \\
\hline Fetal demise & 0 & - & - & - & & - & - & & \\
\hline $\begin{array}{l}\text { Gestational diabetes } \\
\text { mellitus }\end{array}$ & $\begin{array}{l}1 \\
(1.4 \%)\end{array}$ & $\begin{array}{l}0.014 \\
(0.003-0.078)\end{array}$ & $\begin{array}{l}0.99 \\
(0.983-0.994)\end{array}$ & $\begin{array}{l}0.063 \\
(0.011-0.283)\end{array}$ & $\begin{array}{l}0.955 \\
(0.943-0.964)\end{array}$ & $\begin{array}{l}1.4 \\
(0.187-10.432)\end{array}$ & $\begin{array}{l}0.996 \\
(0.967-1.025)\end{array}$ & 0.008 & $p>0.05$ \\
\hline $\begin{array}{l}\text { Total adverse } \\
\text { pregnancy outcome }\end{array}$ & $\begin{array}{l}11 \\
(3.0 \%)\end{array}$ & $\begin{array}{l}0.03 \\
(0.017-0.053)\end{array}$ & \begin{tabular}{|l|}
0.996 \\
$(0.99-0.998)$
\end{tabular} & $\begin{array}{l}0.688 \\
(0.444-0.858)\end{array}$ & $\begin{array}{l}0.764 \\
(0.742-0.785)\end{array}$ & $\begin{array}{l}7.5 \\
(2.426-19.836)\end{array}$ & $\begin{array}{l}0.974 \\
(0.956-0.992)\end{array}$ & 0.108 & $p<0.01$ \\
\hline
\end{tabular}

PPV: Positive predictive value, NPV: Negative predictive value, $+L:+$ Likelihood ratio, $-L:-$ Likelihood ratio, Cl; Confidence interval, $r$ : Correlation coefficient (spearman correlation) 
pregnancy outcomes $(\mathrm{r}=0.189, p<0.01)$ at the cut off level of $\leq 0.50$ c- PAPPA MOM values. It was not statistically significant correlation with oligohydramnios $(\mathrm{r}=-0.001, p>0.05)$ and abruptio placentae $(\mathrm{r}=0.018, p>0.05)$.

The positive likelihood ratio $(+\mathrm{LR})$ of the adverse pregnancy outcomes at the cutoff level of $\leq 0.50$ c-PAPPA MOM values were GHT 1.993 (95\%CI 1.174-3.398), preeclampsia 2.757 (95\%CI 2.01-3.802), preterm birth 1.493 (95\%CI 1.031-2.176), IUGR 2.51 (95\%CI 1.557-4.073), IUEF 2.333 (95\%CI 1.148-4.757), GDM 1.74 (95\%CI 1.148-2.636), total adverse pregnancy outcomes 2.388 (95\%CI $1.889-2.991)$ (Table IV).

ROC curve analysis of c-PAPPA MOM values and adverse pregnancy outcomes were showed in table $\mathrm{V}$. Association between low c-PAPPA MOM values and adverse pregnancy outcomes were showed that preeclampsia was statistically significant and had a fair association (AUC, 0.74 95\% CI 0.690-0.802, $p<0.01)$. Preterm birth was statistically significant but had a fail association (AUC, 0.568 95\% CI $0.512-0.624, p<0.05)$. IUGR was statistically significant and

Table IV: Association with cut off level of $\leq 0.50$ corrected pregnancy-associated plasma protein-A, multiples of median values and adverse pregnancy outcomes

\begin{tabular}{|c|c|c|c|c|c|c|c|c|c|}
\hline & n (1516) & $\begin{array}{l}\text { Sensitivity } \\
95 \% \mathrm{Cl}\end{array}$ & $\begin{array}{l}\text { Specificity } \\
95 \% \mathrm{Cl}\end{array}$ & $\begin{array}{l}\text { PPV } \\
95 \% \mathrm{Cl}\end{array}$ & $\begin{array}{l}\text { NPV } \\
95 \% \mathrm{Cl}\end{array}$ & $\begin{array}{l}+ \text { +LR } \\
95 \% \mathrm{Cl}\end{array}$ & $\begin{array}{l}-\mathrm{LR} \\
95 \% \mathrm{Cl}\end{array}$ & $r$ & $p$ \\
\hline $\begin{array}{l}\text { Gestational } \\
\text { hypertension }\end{array}$ & $\begin{array}{l}10 \\
(30.3 \%)\end{array}$ & $\begin{array}{l}0.303 \\
(0.174-0.473)\end{array}$ & $\begin{array}{l}0.848 \\
(0.829-0.866)\end{array}$ & $\begin{array}{l}0.043 \\
(0.023-0.077)\end{array}$ & $\begin{array}{l}0.982 \\
(0.973-0.988)\end{array}$ & $\begin{array}{l}1.993 \\
(1.174-3.398)\end{array}$ & \begin{tabular}{|l|}
0.822 \\
$(0.655-1.03)$
\end{tabular} & 0.061 & $p<0.05$ \\
\hline Preeclampsia & $\begin{array}{l}27 \\
(22.4 \%)\end{array}$ & $\begin{array}{l}0.397 \\
(0.289-0.516)\end{array}$ & $\begin{array}{l}0.856 \\
(0.837-0.873)\end{array}$ & \begin{tabular}{|l|}
0.115 \\
$(0.08-0.162)$
\end{tabular} & $\begin{array}{l}0.968 \\
(0.957-0.976)\end{array}$ & $\begin{array}{l}2.757 \\
(2.01-3.802)\end{array}$ & $\begin{array}{l}0.704 \\
(0.58-0.855)\end{array}$ & 0.145 & $p<0.01$ \\
\hline Preterm birth & $\begin{array}{l}24 \\
(22.4 \%)\end{array}$ & $\begin{array}{l}0.224 \\
(0.156-0.312)\end{array}$ & $\begin{array}{l}0.85 \\
(0.831-0.868)\end{array}$ & \begin{tabular}{|l|}
0.102 \\
$(0.07-0.147)$
\end{tabular} & $\begin{array}{l}0.935 \\
(0.92-0.947)\end{array}$ & $\begin{array}{l}1.493 \\
(1.031-2.176)\end{array}$ & $\begin{array}{l}0.913 \\
(0.822-1.013)\end{array}$ & 0.053 & $p>0.05$ \\
\hline $\begin{array}{l}\text { Intrauterine growth } \\
\text { restriction }\end{array}$ & $\begin{array}{l}11 \\
(37.9 \%)\end{array}$ & $\begin{array}{l}0.379 \\
(0.227-0.56)\end{array}$ & $\begin{array}{l}0.849 \\
(0.83-0.867)\end{array}$ & $\begin{array}{l}0.047 \\
(0.026-0.082)\end{array}$ & $\begin{array}{l}0.986 \\
(0.978-0.991)\end{array}$ & $\begin{array}{l}2.51 \\
(1.557-4.073)\end{array}$ & $\begin{array}{l}0.731 \\
(0.549-0.972)\end{array}$ & 0.087 & $p>0.05$ \\
\hline Oligohydramnios & $\begin{array}{l}5 \\
(15.2 \%)\end{array}$ & $\begin{array}{l}0.152 \\
(0.067-0.309)\end{array}$ & $\begin{array}{l}0.845 \\
(0.826-0.862)\end{array}$ & $\begin{array}{l}0.021 \\
(0.009-0.049)\end{array}$ & $\begin{array}{l}0.978 \\
(0.969-0.985)\end{array}$ & $\begin{array}{l}0.981 \\
(0.432-2.209)\end{array}$ & $\begin{array}{l}1.004 \\
(0.868-1.162)\end{array}$ & -0.001 & $p<0.05$ \\
\hline Abruptio placentae & $\begin{array}{l}1 \\
(8.3 \%)\end{array}$ & $\begin{array}{l}0.083 \\
(0.015-0.354)\end{array}$ & $\begin{array}{l}0.844 \\
(0.825-0.862)\end{array}$ & $\begin{array}{l}0.004 \\
(0.001-0.024)\end{array}$ & $\begin{array}{l}0.991 \\
(0.985-0.995)\end{array}$ & $\begin{array}{l}0.532 \\
(0.082-3.511)\end{array}$ & $\begin{array}{l}1.086 \\
(0.914-1.289)\end{array}$ & -0.018 & $p<0.05$ \\
\hline Fetal demise & $\begin{array}{l}5 \\
(35.7 \%)\end{array}$ & $\begin{array}{l}0.357 \\
(0.163-0.612)\end{array}$ & $\begin{array}{l}0.847 \\
(0.828-0.864)\end{array}$ & $\begin{array}{l}0.021 \\
(0.009-0.049)\end{array}$ & $\begin{array}{l}0.993 \\
(0.987-0.996)\end{array}$ & $\begin{array}{l}2.333 \\
(1.143-4.757)\end{array}$ & $\begin{array}{l}0.759 \\
(0.513-1.122)\end{array}$ & 0.054 & $p<0.01$ \\
\hline $\begin{array}{l}\text { Gestational diabetes } \\
\text { mellitus }\end{array}$ & $\begin{array}{l}18 \\
(26.1 \%)\end{array}$ & $\begin{array}{l}0.261 \\
(0.172-0.375)\end{array}$ & $\begin{array}{l}0.85 \\
(0.831-0.868)\end{array}$ & $\begin{array}{l}0.077 \\
(0.049-0.118)\end{array}$ & \begin{tabular}{|l|}
0.96 \\
$(0.948-0.97)$
\end{tabular} & $\begin{array}{l}1.74 \\
(1.148-2.636)\end{array}$ & $\begin{array}{l}0.869 \\
(0.755-1.002)\end{array}$ & 0.064 & $p<0.05$ \\
\hline $\begin{array}{l}\text { Total adverse } \\
\text { pregnancy outcome }\end{array}$ & $\begin{array}{l}101 \\
(27.7 \%)\end{array}$ & $\begin{array}{l}0.277 \\
(0.233-0.325)\end{array}$ & $\begin{array}{l}0.884 \\
(0.864-0.901)\end{array}$ & $\begin{array}{l}0.43 \\
(0.368-0.494)\end{array}$ & $\begin{array}{l}0.794 \\
(0.771-815)\end{array}$ & $\begin{array}{l}2.388 \\
(1.889-2.991)\end{array}$ & $\begin{array}{l}0.818 \\
(0.766-0.875)\end{array}$ & 0.189 & $p<0.01$ \\
\hline
\end{tabular}

PPV: Positive predictive value, NPV: Negative predictive value, $+L R:+$ Likelihood ratio, $-L R:$-Likelihood ratio, Cl: Confidence interval, $r$ : Correlation coefficient (spearman correlation)

Table V: Association between low corrected pregnancy-associated plasma protein-A, multiples of median values and adverse pregnancy outcomes

\begin{tabular}{|c|c|c|c|c|c|}
\hline Adverse pregnancy outcomes & $n=1516$ & AUC & $95 \% \mathrm{Cl}$ & & \\
\hline Gestational hypertension & $33(2.2 \%)$ & 0.58 & $0.472-0.688$ & 0.116 & $p>0.05$ \\
\hline Preeclampsia & $68(4.5 \%)$ & 0.746 & $0.690-0.802$ & 0.000 & $p<0.01$ \\
\hline Preterm birth & $107(7.1 \%)$ & 0.568 & $0.512-0.624$ & 0.019 & $p<0.05$ \\
\hline Intrauterine growth restriction & $29(1 ., 9 \%)$ & 0.697 & $0.606-0.787$ & 0.000 & $p<0.01$ \\
\hline Oligohydramnios & $33(2.2 \%)$ & 0.549 & $0.460-0.637$ & 0.338 & $p>0.05$ \\
\hline Abruptio placentae & $12(0.8 \%)$ & 0.470 & $0.323-0.616$ & 0.717 & $p>0.05$ \\
\hline Fetal demise & $14(0.9 \%)$ & 0.657 & $0.528-0.787$ & 0.042 & $p<0.05$ \\
\hline Gestational diabetes mellitus & $69(4.6 \%)$ & 0.571 & $0.499-0.643$ & 0.047 & $p<0.05$ \\
\hline Total adverse pregnancy outcome & $365(24.1 \%)$ & 0.630 & $0.596-0.663$ & 0.000 & $p<0.01$ \\
\hline
\end{tabular}

AUC: Area under curve, Cl: Confidence interval, p: Statistically significant at $<0.05$. ROC: Receiver operating characteristic 
had a fair association (AUC, 0.697, 95\%CI 0.606-0.787, $p<0.01$ ). IUEF was statistically significant but had a poor association (AUC, 0.657 95\%CI 0.528-0.787, $p<0.05$ ). GDM was statistically significant but had a fail association (AUC, 0.571 95\% CI 0.499-0.643, $p<0.05$ ). Total adverse pregnancy outcomes were statistically significant and had a poor association (AUC, 0.630 95\%CI .596-0.663, $p<0.01$ ). GHT was not statistically significant and had a fail association (AUC, 0.58 , 95\% CI $0.472-0.688, p>0.05$ ). Oligohydramnios was not statistically significant and had a fail association (AUC, 0.549, 95\% CI $0.460-0.637, p>0.05$ ). Abruptio placentae had not any statistically significant difference or association (AUC, 0.323$0.616, p>0.05)$.

\section{Discussion}

This study aimed to investigate the relationship between the first-trimester screening maternal serum markers especially corrected PAPPA levels and adverse pregnancy outcomes such as GHT, preeclampsia, IUGR, oligohydramnios, preterm birth, IUEF, GDM, and abruptio placentae (placenta mediated obstetric complications). This study suggests that first-trimester maternal serum low c-PAPPA MOM values were significantly associated with adverse pregnancy outcomes. These markers alone do not have high sensitivity and low false positivity rates for clinical use. However, except for Down syndrome risk assessment in the first-trimester screening test, there was a significantly increased correlation and the risk was identified between adverse pregnancy outcomes and especially low c-PAPPA $(\leq 0.5 \mathrm{MOM})$ values. Our study results agreed with previous studies that have found a low cPAPPA level to be associated with an increased risk of IUGR, preeclampsia, preterm birth, and IUEF (12-18,22,24,25). Similar to previous studies, it was not evidence of statistically significant association with any cut-off levels of corrected free $\beta$-hCG MOM values and other adverse pregnancy outcomes. However, we found that there was a statistically significant association between extreme values of corrected free $\beta$-hCG MOM values and fetal birth weight.

Gomes et al.'s study suggest that an unexplained low level of PAPPA $(<10$ th percentile) during the first trimester can be associated with adverse outcomes and placenta-mediated obstetric complications namely preeclampsia, fetal growth restriction, and abruptio placentae (26). Low levels of free $\beta$ hCG $(<1 \mathrm{st}$, 5th, and 10th percentiles) were not associated with adverse outcomes. Dugoff et al. and Smith et al. studies demonstrated an association between low levels of free $\beta$-hCG $(<0.4$ or $0.5 \mathrm{MOM})$ and birth weight below the 5 th or 10 th percentile (OR 1.6-1.7) (17,23). In our study, we analyzed that below the 5 th percentile of corrected free $\beta$-hCG $(\leq 0.33$ MOM) cut-off level, it was the evidence of statistically significant association with small for gestational age (10th percentile birth weight) $(\mathrm{r}=0.088), p<0.01)$. And also, we found that it was also a statistically significant association with the cutoff level of corrected free $\beta$-hCG $\geq 2.9 \mathrm{MOM}$ values and fetal macrosomia $(>4500$ gram $)(\mathrm{r}=0.068, p<0.01)$.

Smith et al. found that pregnant women who had values below the $5^{\text {th }}$ percentile as a result of their PAPPA measurements between the gestational weeks had an increased risk of IUGR, preterm birth, preeclampsia, and stillbirth $(11,23)$. Based on their findings, they suggested that the control of the IGF system in trophoblasts in the first and early second trimester may play a key role in determining the prognosis of pregnancy (23). In animal experiments, it has been shown that blocking of the PAPPA gene results in a $40 \%$ reduction in fetal development (27). It is thought that PAPPA has either autocrine and paracrine effects on local activities of IGF (28). These data demonstrate an important role of PAPPA in modulating ovarian function, placentation, placental function, and female fertility by control of the bioavailability of ovarian IGF $(8,29)$. If the level of PAPPA is insufficient to successfully cleave IGF, IGF remains in its bound, inactive form, possibly leading to diminished fetal and placental growth and also placental inflammation (11). In our study, we had a statistically significant correlation with IUEF, IUGR, preeclampsia, and low level of PAPPA.

Canini et al. found that PAPPA values were significantly lower in babies with SGA, and PAPPA was significantly higher in babies with LGA (30). They found a significant correlation between birth weight percentiles and c-PAPPA values $(\mathrm{r}=0.192, p<0.001)$. In this case, high PAPPA levels may be expected to be associated with high IGF and thus fetal macrosomia (30). In the study of Dugoff et al., there was no significant association with fetal macrosomia (over $4500 \mathrm{~g}$ ) at levels above the 99.9th percentile of PAPPA level $(p<0.05)(17)$. However, in our study we found that there was a significant correlation between maternal serum free $\beta$-hCG corrected MOM $\geq 2.9$ MOM values with fetal macrosomia infants $(\mathrm{r}=0.068, p<0.01)$. There was no significant association with the cut-off level of free $\beta$-hCG $\leq 0.5 \mathrm{MOM}$ value and GDM $(p>0.05)$. However, it was a significant association between GDM and the cutoff level of corrected PAPPA $\leq 0.5 \mathrm{MOM}$ values $(\mathrm{r}=0.064, p<0.05)$.

The traditional approach to screening for preeclampsia, which is based on maternal demographic characteristics and medical history, identifies approximately $30 \%$ of cases destined to develop early PE for a false-positive rate of 5\% (26). It is necessary to find additional predictive parameters to classify the high-risk pregnancy group. Poon et al used the combination of mean arterial pressure (MAP), placental growth factor (PIGF), and PAPPA MOM values to increase the likelihood ratio or post-test probability. In this way, they were aimed at the care of low-risk pregnancies is decentralized to local, 1-stop settings or at home, whereas high-risk pregnancies are cared for in specialized centers (31).

We were referred to analyze the predictive values of ad- 
verse pregnancy outcomes by likelihood ratio because it can be used to calculate the post-test probability and it is less affected by the prevalence of the adverse pregnancy outcome (Table III and IV). This situation provided us that if we detected increased post-test probability, we would have guided the patient to another test and the first test's post-probability value could be used as the next test pre-test probability. In this way, calculating the final likelihood ratio of certain cutoff levels obtained from a meta-analysis may be a useful tool with additional tests for conditions and diseases that have low prevalence. In our study, we have reported the likelihood ratio results of PAPPA $\leq 0.25$ and $\leq 0.50$ MOM cutoff values to be able to use in future meta-analysis. And we saw that the positive likelihood ratio changes by the cut of level value are certain these changes will reflect the post-test probability. Posttest probability calculating is very important nowadays because we are living in the artificial intelligence century. In artificial intelligence, one of the suitable and calculable data that can be used in artificial intelligence algorithms may be the post-test probability value. According to Bayes theorem, the formula may be as shown (32).

Post-test probability of total adverse pregnancy outcome for first pregnancy $=$ pre-test probability (i.e. prevalence) $\times$ positive likelihood ratio (i.e. first-trimester screening test) $\times$ positive likelihood ratio (Doppler ultrasonography) $\times$ positive likelihood ratio (i.e. second-trimester screening test) $\times$ positive likelihood ratio (second-trimester ultrasonography)

Taking into account the cutoff level of the test and considering the patient in a high-risk group or not is insufficient to artificial intelligence algorithms. Such a formula as shown may help the calculation of the pre-test probability of the firsttrimester screening test of the second pregnancy and may be useful data for artificial intelligence algorithms to identify and follow the high-risk pregnant women in the National health care program.

The most important limitation of our study is the need for more cases in the evaluation of the prediction of adverse obstetric outcomes with low prevalence. It may not be appropriate to exclude miscarriage cases because of the significant association between the low levels of PAPPA and adverse pregnancy outcomes such as IUGR and IUEF. While we were preparing this article for an academic publication, the military coup incident took place. We could not access the data after 2009 due to the hospitals' handover and system changes. It may be appropriate to evaluate the association between the low level of PAPPA and miscarriages in further studies in the future.

\section{Conclusion}

In our study, it was observed that first-trimester maternal serum low PAPPA MOM values were significantly associated with adverse pregnancy outcomes. These markers alone do not have high sensitivity and low false positivity rates for clinical use. However, except for Down syndrome risk assessment in the first-trimester screening test, there was a significantly increased correlation, and the risk was identified between adverse pregnancy outcomes and especially low corrected PAPPA $(\leq 0.5 \mathrm{MOM})$ values. It is necessary to be careful when planning the follow-up of pregnancies of first-trimester markers by increasing the sensitivity and decreasing the false positivity with additional tests to be performed in the following gestational weeks.

Ethics approval and consent to participate: All participants signed informed written consent before being enrolled in the study. The study was reviewed and approved by the ethics committee of GATA Haydarpasa Education Hospital. All procedures were performed according to the Declaration of Helsinki.

Availability of data and materials: The data supporting this study is available through the corresponding author upon reasonable request.

Competing interests: The authors declare that they have no competing interests.

Funding: There is no funding or financial support used for this study.

Authors' contributions: GG: Project development, data collection, analysis, interpretation of data, manuscript writing, revising the manuscript. YKA: Manuscript writing and revising the manuscript. MM: Idea, check, and consultancy. All authors contributed to the writing of the paper, and have read and approved the final manuscript.

Acknowledgment: None

\section{References}

1. Lin TM, Galbert SP, Kiefer D, Spellacy WN, Gall S. Characterization of four human pregnancy-associated plasma proteins. Am J Obstet Gynecol. 1974;118(2):22336. Doi: 10.1016/0002-9378(74)90553-5.

2. Conover CA, Kiefer MC, Zapf J. Posttranslational regulation of insulin-like growth factor binding protein-4 in normal and transformed human fibroblasts. Insulin-like growth factor dependence and biological studies. J Clin Invest. 1993;91(3):1129-37. Doi: 10.1172/JCI116272.

3. Wald NJ, Kennard A, Densem JW, Cuckle HS, Chard T, Butler L. Antenatal maternal serum screening for Down's syndrome: results of a demonstration project. BMJ. 1992;305(6850):391-4. Doi: 10.1136/bmj.305.6850.391.

4. Wald NJ, Densem JW, Smith D, Klee GG. Four-marker serum screening for Down's syndrome. Prenat Diagn. 1994;14(8):707-16. Doi: 10.1002/pd.1970140810.

5. Reynolds TM, Penney MD, Hughes H, John R. The Effect of Weight Correction on Risk Calculations for Down's Syndrome Screening. Ann Clin Biochem. 1991;28(Pt 3):245-9. Doi: 10.1177/000456329102800308.

6. Clemmons DR. Role of insulin-like growth factor binding 
proteins in controlling IGF actions. Molecular and cellular endocrinology. Mol Cell Endocrinol. 1998;140(1-2):1924.

7. Jones JI, Clemmons DR. Insulin-like growth factors and their binding proteins: biological actions. Endocr Rev. 1995;16(1):3-34. Doi: 10.1210/edrv-16-1-3.

8. Nyegaard M, Overgaard MT, Su YQ, Hamilton AE, Kwintkiewicz J, Hsieh M, et al. Lack of functional pregnancy-associated plasma protein-A (PAPPA) compromises mouse ovarian steroidogenesis and female fertility. Biol Reprod. 2010;82(6):1129-38. Doi: 10.1095/biolreprod.109.079517.

9. Cataldo NA, Giudice LC. Insulin-like growth factor binding protein profiles in human ovarian follicular fluid correlate with follicular functional status. J Clin Endocrinol Metab. 1992;74(4):821-9. Doi: 10.1210/jcem.74.4. 1372322.

10. Sun IY, Overgaard MT, Oxvig C, Giudice LC. Pregnancyassociated plasma protein A proteolytic activity is associated with the human placental trophoblast cell membrane. J Clin Endocrinol Metab. 2002;87(11):5235-40. Doi: 10.1210/jc.2002-020561.

11. Peterson SE, Simhan HN. First-trimester pregnancy-associated plasma protein A and subsequent abnormalities of fetal growth. Am J Obstet Gynecol. 2008;198(5):e43-5. Doi: 10.1016/j.ajog.2007.12.026. Epub 2008 Mar 4.

12. Irwin JC, Suen LF, Martina NA, Mark SP. Giudice LC. Role of the IGF system in trophoblast invasion and preeclampsia. Hum Reprod. 1999;14 Suppl 2:90-6. Doi: 10.1093/humrep/14.suppl_2.90.

13. Spencer K, Cowans NJ, Avgidou K, Molina F, Nicolaides KH. First-trimester biochemical markers of aneuploidy and the prediction of small-for-gestational age fetuses. Ultrasound Obstet Gynecol. 2008;31(1):15-9. Doi: 10. 1002/uog.5165.

14. Spencer K, Cowans NJ, Molina F, Kagan KO, Nicolaides KH. First-trimester ultrasound and biochemical markers of aneuploidy and the prediction of preterm or early preterm delivery. Ultrasound Obstet Gynecol. 2008; 31(2):147-52. Doi: 10.1002/uog.5163.

15. Spencer K, Cowans NJ, Nicolaides KH. Low levels of maternal serum PAPP-A in the first trimester and the risk of pre-eclampsia. Prenat Diagn. 2008;28(1):7-10. Doi: 10.1002/pd.1890.

16. Spencer K. Cowans NJ. Avgidou K. Nicolaides KH. Firsttrimester ultrasound and biochemical markers of aneuploidy and the prediction of impending fetal death. Ultrasound Obstet Gynecol. 2006;28(5):637-43. Doi: 10.1002/uog.3809.

17. Dugoff L, Hobbins JC, Malone FD, Porter TF, Luthy D, Comstock $\mathrm{CH}$, et al. First-trimester maternal serum PAPP-A and free-beta subunit human chorionic gonadotropin concentrations and nuchal translucency are associated with obstetric complications: a population-based screening study (the FASTER Trial). Am J Obstet Gynecol. 2004;191(4):1446-51. Doi: 10.1016/j.ajog. 2004.06.052.

18. Goetzl L, Krantz D, Simpson JL, Silver RK, Zachary JM, Pergament E, et al. Pregnancy-associated plasma protein A, free beta-hCG, nuchal translucency, and risk of pregnancy loss. Obstet Gynecol. 2004;104(1):30-6. Doi: 10.1097/01.AOG.0000129969.78308.4f.

19. Spencer K, Yu CK, Cowans NJ, Otigbah C, Nicolaides $\mathrm{KH}$. Prediction of pregnancy complications by firsttrimester maternal serum PAPP-A and free beta-hCG and with second-trimester uterine artery Doppler. Prenat Diagn. 2005;25(10):949-53. Doi: 10.1002/pd.1251.

20. Barrett SL, Bower C, Hadlow NC. Use of the combined first-trimester screen result and low PAPP-A to predict risk of adverse fetal outcomes. Prenat Diagn. 2008;28 (1):28-35. Doi: 10.1002/pd.1898.

21. Yaron Y, Ochshorn Y, Heifetz S, Lehavi O, Sapir Y, OrrUrtreger A. First trimester maternal serum free human chorionic gonadotropin as a predictor of adverse pregnancy outcome. Fetal Diagn Ther. 2002;17(6):352-6. Doi: $10.1159 / 000065384$.

22. Krantz D, Goetzl L, Simpson JL, Thom E, Zachary J, Hallahan TW, et al. Association of extreme first-trimester free human chorionic gonadotropin-beta, pregnancy-associated plasma protein A, and nuchal translucency with intrauterine growth restriction and other adverse pregnancy outcomes. Am J Obstet Gynecol. 2004;191(4):1452-8. Doi: 10.1016/j.ajog.2004.05.068.

23. Smith GC, Stenhouse EJ, Crossley JA, Aitken DA, Cameron AD, Connor JM. Early pregnancy levels of pregnancy-associated plasma protein a and the risk of intrauterine growth restriction, premature birth, preeclampsia, and stillbirth. J Clin Endocrinol Metab. 2002; 87(4):1762-7. Doi: 10.1210/jcem.87.4.8430.

24. Spencer K. Second-trimester prenatal screening for Down syndrome and the relationship of maternal serum biochemical markers to pregnancy complications with adverse outcome. Prenat Diagn. 2000;20(8):652-6.

25. Cohen JL, Smilen KE, Bianco AT, Moshier EL, Ferrara LA, Stone JL. Predictive value of combined serum biomarkers for adverse pregnancy outcomes. Eur J Obstet Gynecol Reprod Biol. 2014;181:89-94. Doi: 10.1016/ j.ejogrb.2014.07.018.

26. Gomes MS, Carlos-Alves M, Trocado V, Arteiro D, Pinheiro P. Prediction of adverse pregnancy outcomes by extreme values of first trimester screening markers. Obstet Med. 2017;10(3):132-137. Doi:10.1177/1753495X177 04799.

27. Kniss DA, Shubert PJ, Zimmerman PD, Landon MB, Gabbe SG. Insulinlike growth factors. Their regulation of glucose and amino acid transport in placental trophoblasts isolated from first-trimester chorionic villi. J Reprod Med. 1994;39(4):249-56. 
28. Giudice LC. Insulin-like growth factor family in Graafian follicle development and function. J Soc Gynecol Investig. 2001;8(1 Suppl Proceedings):S26-9.

29. Conover CA, Bale LK, Overgaard MT, Johnstone EW, Laursen UH, Fuchtbauer EM, et al. Metalloproteinase pregnancy-associated plasma protein A is a critical growth regulatory factor during fetal development. Development. 2004;131(5):1187-94. Doi: 10.1242/dev.00997.

30. Canini S, Prefumo F, Pastorino D, Crocetti L, Afflitto CG, Venturini PL, et al. Association between birth weight and first-trimester free beta-human chorionic gonadotropin and pregnancy-associated plasma protein A. Fertil Steril. 2008;89(1):174-8. Doi: 10.1016/j.fertnstert.2007.02.024.

31. Poon LC, Kametas NA, Maiz N, Akolekar R, Nicolaides KH. First-trimester prediction of hypertensive disorders in pregnancy. Hypertension. 2009;53(5):812-8. Doi: 10.1161/HYPERTENSIONAHA.108.127977.

32. Theorem B. Calculate the probability of an outcome based on clinical conditions and diagnostic tests. Available from: https://www.scalelive.com/bayes-theorem.html. 